\title{
$\beta$-Radioluminescence Imaging: A Comparative Evaluation with Cerenkov Luminescence Imaging
}

\author{
Martin T. King ${ }^{1}$, Colin M. Carpenter ${ }^{1}$, Conroy Sun ${ }^{2}$, Xiaowei Ma ${ }^{3,4}$, Quynh-Thu Le ${ }^{1}$, John B. Sunwoo ${ }^{5}$ Zhen Cheng ${ }^{3}$, \\ Guillem Pratx ${ }^{1}$, and Lei Xing ${ }^{1}$ \\ ${ }^{I}$ Department of Radiation Oncology, Stanford University, Stanford, California; ${ }^{2}$ College of Pharmacy, Oregon State University, \\ Portland, Oregon; ${ }^{3}$ Department of Radiology, Stanford University, Stanford, California; ${ }^{4}$ Department of Nuclear Medicine, Xijing \\ Hospital, Fourth Military Medical University, Xi'an, Shaanxi, China; and ${ }^{5}$ Department of Otolaryngology, Stanford University, \\ Stanford, California
}

\begin{abstract}
Cerenkov luminescence imaging (CLI) can provide high-resolution images of ${ }^{18} \mathrm{~F}-\mathrm{FDG}$-avid tumors but requires prolonged acquisition times because of low photon sensitivity. In this study, we proposed a new modality, termed $\beta$-radioluminescence imaging ( $\beta$-RLI), which incorporates a scintillator with a $y$-rejection strategy for imaging $\beta$ particles. We performed a comparative evaluation of $\beta$-RLI with CLI in both in vitro and in vivo systems. Methods: Using in vitro phantoms, we characterized the photon sensitivity and resolution of CLI and $\beta$-RLI. We also conducted a series of in vivo experiments with xenograft mouse models using both amelanotic (A375, UMSCC1Luc) and melanotic (B16F10-Luc) cell lines. The B16F10 and UMSCC1 cell lines were transfected with the luciferase gene (Luc). CLI was acquired over $300 \mathrm{~s}$, and $\beta$-RLI was acquired using two 10-s acquisitions. We correlated ${ }^{18} \mathrm{~F}-\mathrm{FDG}$ activities, as assessed by PET, with tumor radiances for both $\beta-\mathrm{RLI}$ and $\mathrm{CLI}$. We also compared tumor signal-to-background ratios (SBRs) between these modalities for amelanotic and melanotic tumors. Results: For in vitro experiments, the photon sensitivity for $\beta$-RLI was 560 -fold greater than that for CLI. However, the spatial resolution for $\beta$-RLI $(4.4 \mathrm{~mm})$ was inferior to that of CLI $(1.0 \mathrm{~mm})$. For in vivo experiments, correlations between ${ }^{18} \mathrm{~F}-\mathrm{FDG}$ activity and tumor radiance were $0.52(P<0.01)$ for $\beta-\mathrm{RLI}$, $0.81(P=0.01)$ for amelanotic lesions with $\mathrm{CLI}$, and -0.08 (negative contrast; $P=0.80$ ) for melanotic lesions with CLI. Nine of 13 melanotic lesions had an SBR less than 1 for CLI, despite an SBR greater than 1 among all lesions for $\beta$-RLI. Conclusion: $\beta$-RLI can produce functional images of both amelanotic and melanotic tumors in a shorter time frame than CLI. Further engineering developments are needed to realize the full clinical potential of this modality.
\end{abstract}

Key Words: beta; Cerenkov; radioluminescence; melanoma; surgery

J Nucl Med 2015; 56:1458-1464

DOI: 10.2967/jnumed.115.158337

O erenkov luminescence imaging (CLI) is an optical radionuclide imaging technique that can produce high-resolution (1-2 mm) functional images of ${ }^{18} \mathrm{~F}(1-3)$. Because CLI signals demonstrate

Received Mar. 26, 2015; revision accepted Jul. 8, 2015.

For correspondence or reprints contact: Martin T. King, Department of Radiation Oncology, Stanford University School of Medicine, 875 Blake Wilbur Dr., Stanford, CA 94305.

E-mail: mtking12@gmail.com

Published online Jul. 23, 2015.

COPYRIGHT (C) 2015 by the Society of Nuclear Medicine and Molecular Imaging, Inc. a strong linear relationship with PET activity (3), this technology can be used for measuring tumor burden after chemotherapy administration $(4,5)$ and surgical resection $(6,7)$. Many radiotracers including ${ }^{18} \mathrm{~F}-\mathrm{FDG}$ are already approved by the Federal Drug Administration. As a result, CLI has immediate translational potential, and studies have already been reported in human patients $(8-10)$.

However, CLI is limited by its relatively low photon sensitivity. Investigators have estimated that a $0.635-\mathrm{MeV}$ positron from ${ }^{18} \mathrm{~F}$ can produce only 20 photons with wavelengths between 250 and $600 \mathrm{~nm}(1)$. As a result, prolonged acquisition times on the order of 3-5 min in a darkened environment are required $(3,7,9)$. These stringent acquisition conditions may be difficult to satisfy in certain clinical settings, including the operating room.

One potential strategy for improving photon sensitivity is to use a scintillator, which can convert $\beta$ particles and $\gamma$ rays from ${ }^{18} \mathrm{~F}$ into optical light. Scintillators can produce between 1,000 and more than 100,000 photons per $\mathrm{MeV}$, depending on the material used (11). Scintillators have been incorporated into handheld radioactive probes and cameras for radio-guided surgical applications (12-16).

In recent years, non-Cerenkov optical radionuclide imaging techniques, termed radioluminescence imaging (RLI), have been evaluated (17). In particular, investigators have performed in vitro and in vivo imaging of the pure $\gamma$ emitter ${ }^{99 \mathrm{~m}} \mathrm{Tc}$ by placing bismuth germanate oxide scintillator crystals between the radioactive source and the camera within a commercial small-animal imaging system. The system resolution was $1.3 \mathrm{~mm}$, and a collimator was present (18). Non-scintillator-based RLI experiments for ${ }^{99 \mathrm{~m} T c}$ (19) and an $\alpha$ emitter (20) have also been reported. In our group, 1 investigator performed RLI of ${ }^{18} \mathrm{~F}-\mathrm{FDG}$-uptake in single cells by imaging a radioactive cell monolayer in direct contact with a scintillator using microscopy (21). Another investigator designed a fiber-optic system for imaging ${ }^{18} \mathrm{~F}$-FDG from ex vivo atherosclerotic plaques that were covered by a scintillator (22).

In this study, we proposed a new method for imaging $\beta$ particles from ${ }^{18} \mathrm{~F}$ using a scintillator. This method, termed $\beta$-radioluminescence imaging $(\beta-R L I)$, incorporates RLI with a $\gamma$-rejection strategy adapted from $\beta$ probes (12). We hypothesized that $\beta$-RLI may provide enhanced photon sensitivity, compared with CLI. Furthermore, $\beta$-RLI may produce images of intact ${ }^{18} \mathrm{~F}-\mathrm{FDG}$-avid tumors in a shorter time frame than that required with CLI. The purpose of this study was to perform a comparative evaluation of $\beta$-RLI with CLI in both in vitro and in vivo settings. 
TABLE 1

Components Required for Acquiring CLI, RLI, and Block-RLI

\begin{tabular}{llll}
\hline \multicolumn{1}{c}{ Component } & CLI & RLI & Block-RLI \\
\hline Charge-coupled device camera & Yes & Yes & Yes \\
Scintillator & No & Yes & Yes \\
Steel & No & No & Yes \\
Object & Yes & Yes & Yes \\
Exposure time (s) & & & \\
$\quad$ In vitro & $30-180$ & $10-60$ & $10-60$ \\
\hline In vivo & 300 & 10 & 10 \\
\hline
\end{tabular}

Scintillator and steel plates were placed directly above object. $\beta$-RLIs were computed by subtracting block-RLI from RLI.

\section{MATERIALS AND METHODS}

\section{Materials}

${ }^{18}$ F-FDG was produced from the radiochemistry facility (Stanford, CA). Two melanoma cell lines, melanotic B16F10 and amelanotic A375, were obtained from the American Type Culture Collection. The head and neck UMSCC1 cell line was obtained from the University of Michigan. The B16F10 and UMSCC1 cell lines were transfected with the pGL4.10[luc2] vector from Promega, using the jetPRIME transfection reagent (Polyplus) to create B16F10-Luc and UMSCC1Luc cell lines.

\section{Optimal Imaging Modalities}

All images were acquired using an IVIS system (200 or Spectrum) with medium binning and a 10- to 13-cm imaging field of view. Essential acquisition parameters for all imaging modalities are listed in Table 1. A schematic for $\beta$-RLI is shown in Figure 1.

Optical imaging modalities are described as follows. For CLI, no material was placed between the camera and the object of interest. For RLI, a scintillator was positioned approximately $1 \mathrm{~mm}$ above the object but below the camera. Optical signals from both $\beta$ particles and $\gamma$ rays were collected.

$$
\operatorname{RLI}=\beta+\gamma .
$$

Eq. 1

For block-RLI, a 1-mm-thick stainless steel slab (Evansville Sheet Metal Works) was placed between the scintillator and the object. Stainless steel was used because of its ability to block almost all $\beta$ particles with

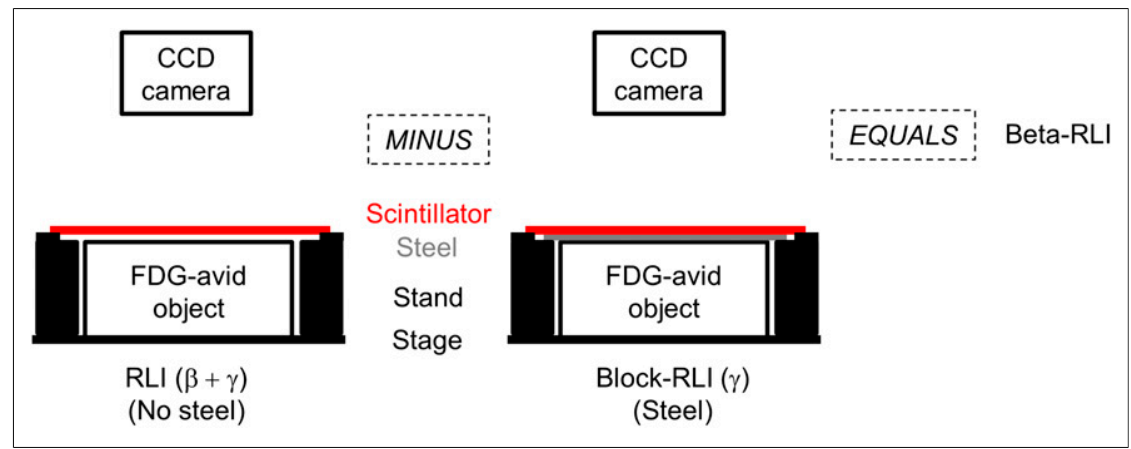

FIGURE 1. $\beta-R L I$ acquisition schematic. $\beta-R L I$ was acquired by obtaining difference between $\mathrm{RLI}$ (containing both $\beta$ particles and y rays ) and block-RLI (containing mostly y rays). Schematic is not drawn to scale. Scintillator thickness and stainless steel thickness were less than 1 and $1 \mathrm{~mm}$, respectively. $\mathrm{CCD}=$ charge-coupled device; $\mathrm{RLI}=$ radioluminescence image. energies less than $1.5 \mathrm{MeV}$ (12). The optical signal captured by the camera can be expressed as:

$$
\text { Block-RLI }=\alpha_{1} \beta+\alpha_{2} \gamma
$$

where $\alpha_{1}$ and $\alpha_{2}$ are the percentage $\beta$ and $\gamma$ transmissions through stainless steel. For this study, estimates of $\alpha_{1}$ and $\alpha_{2}$ were 0.03 and 0.91 , respectively (supplemental data; supplemental materials are available at http://jnm.snmjournals.org). For $\beta$-RLI (with $\gamma$ rejection), this image was calculated as the difference between the RLI and block-RLI, which was multiplied by $1 / \alpha_{2}$ to eliminate the $\gamma$ component.

$$
\beta-\mathrm{RLI}=\mathrm{RLI}-1 / \alpha_{2} \times \text { block-RLI }=\beta \times\left(1-\alpha_{1} / \alpha_{2}\right) . \quad \text { Eq. } 3
$$

All RLI and block-RLI were acquired using a radioisotopic screen (Bruker Biosciences Corp.), although other scintillators were also evaluated (supplemental data).

\section{Optical Image Processing}

Median filtration (3-pixel width) was applied to CLI, RLI, and blockRLI. RLI and block-RLI also underwent additional gaussian smoothing $(\sigma=2 \mathrm{~mm})$ to reduce noise. Bias correction, flattening field correction, and cosmic ray correction (median filtration of pixel values more than 10 SDs from the image mean) were also applied. All processing was performed using software written in Python 3.2.3 (Python Software Foundation).

\section{In Vitro}

Signal Uniformity. The outer shell of the Micro Deluxe Phantom (Data Spectrum Corp.) was filled with $20 \mathrm{~mL}$ of $1 \%$ agarose (SigmaAldrich Chemical Co.) to create a uniform base. A $30-\mathrm{mL}$ solution of $5.1 \mathrm{MBq}(137.5 \mu \mathrm{Ci})$ of ${ }^{18} \mathrm{~F}-\mathrm{FDG}(254 \mathrm{kBq} / \mathrm{mL}$ or $6.9 \mu \mathrm{Ci} / \mathrm{mL})$ was prepared. RLI, block-RLI, $\beta$-RLI, and CLI were obtained. Profiles across images were extracted. An additional experiment evaluating signal uniformity over a nonuniform background was also conducted (supplemental data).

Resolution. A 0.5 -mm internal diameter capillary tube (VitroCom) was filled with ${ }^{18} \mathrm{~F}$-FDG $(370 \mathrm{kBq} / \mathrm{mL}$ or $10 \mu \mathrm{Ci} / \mathrm{mL}) . \beta$-RLI and CLI were acquired with the scintillator placed directly against the capillary tube. Small binning was used. Profiles were drawn perpendicular to the capillary tube, and full-width half maximum (FWHM) values were extracted.

Object-Scintillator Distance Dependencies for $\beta$-RLI. A 0.5-mmdiameter capillary tube was filled with $259 \mathrm{kBq} / \mathrm{mL}(7 \mu \mathrm{Ci} / \mathrm{mL})$ of ${ }^{18} \mathrm{~F}-\mathrm{FDG}$ and positioned on a stack of ten $1-\mathrm{mm}$-thick acrylic slabs. Serial $\beta$-RLI was acquired at distances between the capillary tube and scintillator of 1-10 mm. FWHM values and maximum signal ratios (maximal signal at specified distance/maximal signal at 1-mm distance for air) from profiles drawn perpendicular to the capillary tube were acquired for these air distances. To evaluate depth dependency, maximum signal ratios were also acquired for a capillary tube placed under 1-3-mm of acrylic.

Photon Sensitivity Analysis. Serial 10-fold dilutions of ${ }^{18} \mathrm{~F}$-FDG droplets $(370 \mathrm{kBq}$ [10 $\mu \mathrm{Ci}]$ to $0.037 \mathrm{kBq}[0.001 \mu \mathrm{Ci}])$ dissolved in $50 \mu \mathrm{L}$ of Matrigel (BD Biosciences) were prepared on microscope cover glasses (Fisher Scientific) in triplicate. Final concentrations ranged from $7.4 \mathrm{MBq} / \mathrm{mL}(200 \mu \mathrm{Ci} / \mathrm{mL})$ to $0.74 \mathrm{kBq} / \mathrm{mL}(0.02 \mu \mathrm{Ci} / \mathrm{mL})$. CLI and $\beta$-RLI were acquired. The average intensities of regions of interests (ROIs) over the droplets 
were plotted against ${ }^{18} \mathrm{~F}-\mathrm{FDG}$ activities. The slopes of the linear regression curves represented the photon sensitivities for each modality. A similar analysis involving serial dilutions of ${ }^{18} \mathrm{~F}-\mathrm{FDG}$-avid B16F10 cells is described in the supplemental data.

\section{In Vivo}

Tumor Model. All animal studies were conducted in accordance with the institutional Administrative Panel on Laboratory Animal Care. B16F10-Luc, A375, and UMSCC1-Luc cells were cultured in Dulbecco modified Eagle medium supplemented with $10 \%$ fetal bovine serum and $1 \%$ penicillin-streptomycin (Invitrogen Life Technologies) at $37^{\circ} \mathrm{C}$. Approximately $1.0 \mathrm{E} 6$ cells suspended in phosphate-buffered saline were inoculated into the right flank of female athymic nude mice (Charles River Laboratories) at 6-8 wk old. Mice were imaged after 9-10 d for B16F10-Luc and A375 cells and after 5-6 wk for UMSCC1-Luc cells.

PET Imaging. PET images were acquired using a microPET/CT scanner (Inveon; Siemens Medical Solutions USA, Inc.). Tumorbearing animals were injected with $11.1-14.8 \mathrm{MBq}(300-400 \mu \mathrm{Ci})$ of ${ }^{18} \mathrm{~F}$-FDG dissolved in $150 \mu \mathrm{L}$ of phosphate-buffered saline via the tail vein. After 30-60 min, mice were anesthetized with $2 \%$ isoflurane (Aerrane' Baxter), positioned prone, and imaged. PET images were reconstructed using the ordered-subsets expectation maximum algorithm with attenuation correction if CT data were available. Tumor activities were quantified using commercial software.

Optical Imaging. Acquisition times were $300 \mathrm{~s}$ for CLI and $10 \mathrm{~s}$ for both RLI and block-RLI. For CLI, 1-3 mice were imaged at the same time. For $\beta$-RLI, 1 mouse was imaged each time. BLI was acquired over $60 \mathrm{~s}$ at $10 \mathrm{~min}$ after intraperitoneal injection of D-Luciferin $(150 \mathrm{mg} / \mathrm{kg})$.

For the melanotic B16F10-Luc $(n=14)$ cell line, after tumor exposure (skin excision), $\beta$-RLI and CLI were acquired. Given negative tumor contrast with CLI, BLI was performed and mice were sacrificed. After confirming that photons from BLI did not pass through the scintillator (data not shown), $\beta$-RLI was performed after partial and full resections for 5 and 10 mice, respectively. After full resection, BLI was acquired for evaluating the presence of residual disease. CLI was not performed after partial and full resections because of the presence of D-Luciferin.

For the amelanotic A375 $(n=5)$ cell line, $\beta$-RLI and CLI were acquired after tumor exposure, partial resection, and full resection. Mice were not repositioned between $\beta$-RLI and CLI. Mice were sacrificed after initial imaging of the exposed tumors.

For the amelanotic UMSCC1-Luc $(n=13)$ cell line, after tumor exposure, $\beta$-RLI and CLI were acquired for 13 and 4 mice, respectively. Mice were then sacrificed.

\section{Tumor Signal-to-Background Ratio (SBR)}

Tumor ROIs were delineated with white light and functional (CLI, RLI, $\beta$-RLI, and BLI) images using ITK-SNAP 3.0 software
(ITK-SNAP) (23). Background ROIs were drawn around the tumor ROI using a circular tool with a diameter of 4-6 mm. However, regions covering intact skin were excluded. Tumor SBRs were then computed.

\section{Statistical Analysis}

The following analyses were conducted: Pearson correlation coefficients between ${ }^{18} \mathrm{~F}$-FDG PET activity (scaled to the time of optical imaging) and tumor radiance after tumor exposure were calculated for $\beta$-RLI, CLI for amelanotic tumors, and CLI for melanotic tumors.

Pearson correlation coefficients for SBRs from CLI versus $\beta$-RLI were calculated for amelanotic and melanotic tumors. A comparative analysis for SBR values from $\beta$-RLI versus RLI was also conducted (supplemental data).

One-sided $t$ tests were conducted to determine whether tumor SBR declines between successive stages of serial resection were statistically significant. Tests were used for CLI and $\beta$-RLI of A375 tumors $(n=5)$ and for $\beta$-RLI of B16F10-Luc tumors $(n=5)$.

Among the grossly resected B16F10-Luc tumors with residual disease, as visualized with BLI, the median SBR and the percentage of cases with an SBR greater than 1.2 were tabulated.

\section{RESULTS}

\section{In Vitro Experiments}

Figure 2 shows RLI, block-RLI, $\beta$-RLI, and CLI of the cylinder with ${ }^{18}$ F-FDG. As exhibited in the profiles, the elevated background in RLI was suppressed in $\beta$-RLI. However, the resulting $\beta$-RLI was not nearly as uniform as CLI. In another experiment, $\beta$-RLI was effective in suppressing $\gamma$ rays from a nonuniform background (supplemental data).

As shown in Figure 3A, maximum signal ratios degraded as the air distance between the scintillator and the capillary tube increased. Furthermore, marked declines in maximum signal ratios were observed at 1- to 3-mm depths below the acrylic slabs. With respect to resolution, the FWHM values of profiles perpendicular to the $0.5-\mathrm{mm}$ capillary source were $1.0 \mathrm{~mm}$ for CLI and $4.4 \mathrm{~mm}$ for $\beta$-RLI when the scintillator was placed against the capillary tube. Figure 3B shows that the $\beta$-RLI resolution decreased as the distance between the scintillator and the capillary tube increased. Figure $3 \mathrm{C}$ depicts intensity values for serial ${ }^{18} \mathrm{~F}$-FDG dilutions. Photon sensitivity values for $\beta$-RLI and CLI were $391.8 \mathrm{p} / \mathrm{s} / \mathrm{cm}^{2} / \mathrm{sr} / \mathrm{Bq}(37.3$ counts per second [cps] $/ \mathrm{kBq})$ and $0.7 \mathrm{p} / \mathrm{s} / \mathrm{cm}^{2} / \mathrm{sr} / \mathrm{Bq}(0.07 \mathrm{cps} / \mathrm{kBq})$, respectively. $\beta$-RLI was 560 times more sensitive than CLI based on this experiment. $\beta-R L I$ also exhibited greater photon sensitivity in imaging B16F10 cells (supplemental data).

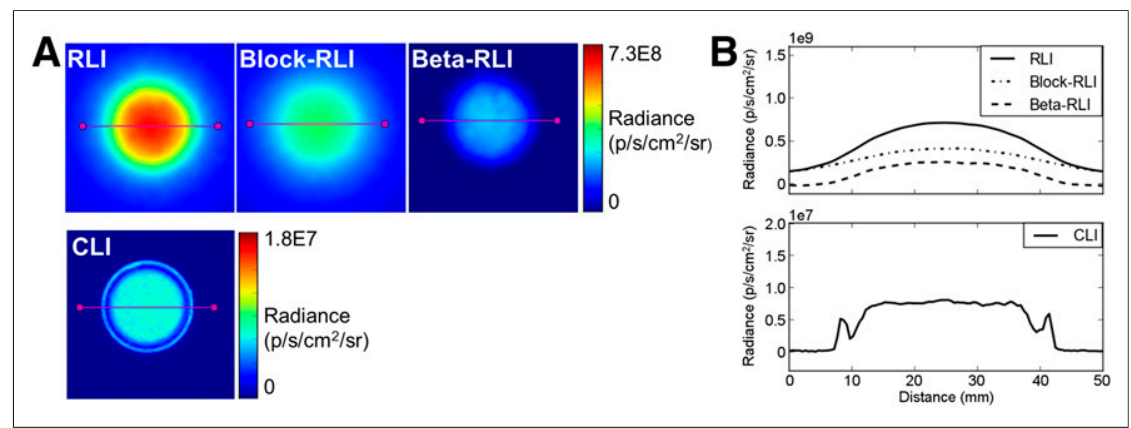

FIGURE 2. Signal uniformity across cylindric phantom filled with $5.1 \mathrm{MBq}(137.5 \mu \mathrm{Ci})$ of ${ }^{18} \mathrm{~F}-\mathrm{FDG}$. (A) Optical images (RLI, block-RLI, $\beta$-RLI, CLI). Intensity values for profile analysis were extracted from purple lines across phantom. (B) Intensity profiles.

\section{In Vivo Studies}

Tumor Exposure. Figure 4A shows the relationship between preresection tumor radiance and ${ }^{18} \mathrm{~F}-\mathrm{FDG}$ activity $(\mathrm{kBq} / \mathrm{mL})$ for $\beta$-RLI, CLI for amelanotic lesions, and CLI for melanotic lesions. The correlation coefficients between radiance and ${ }^{18} \mathrm{~F}$-FDG activity were $0.52(P<$ $0.01)$ for $\beta$-RLI, $0.81(P=0.01)$ for amelanotic lesions with CLI and -0.08 $(P=0.80)$ for melanotic lesions with CLI. Figure 4B shows the relationships between SBR values between CLI and $\beta$ RLI. Correlation coefficients were 0.57 $(P=0.11)$ for amelanotic lesions and 0.55 


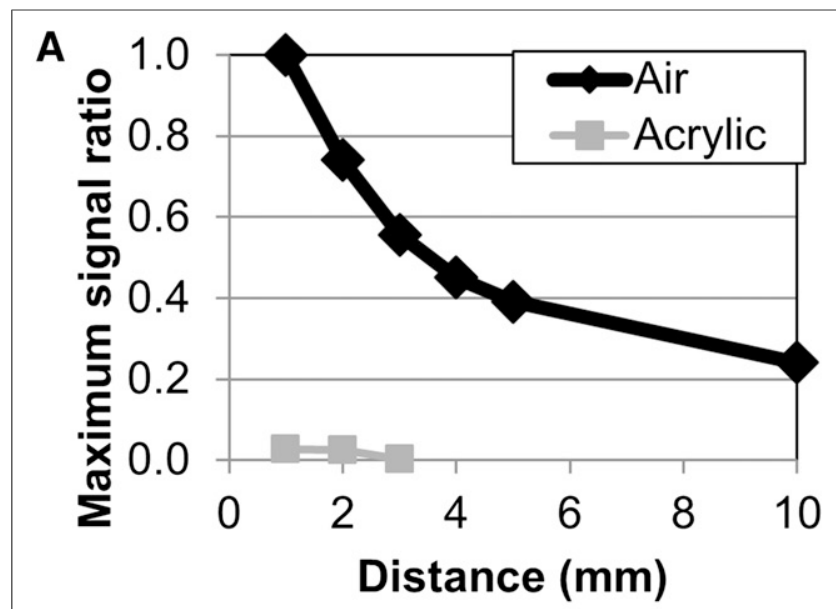

B

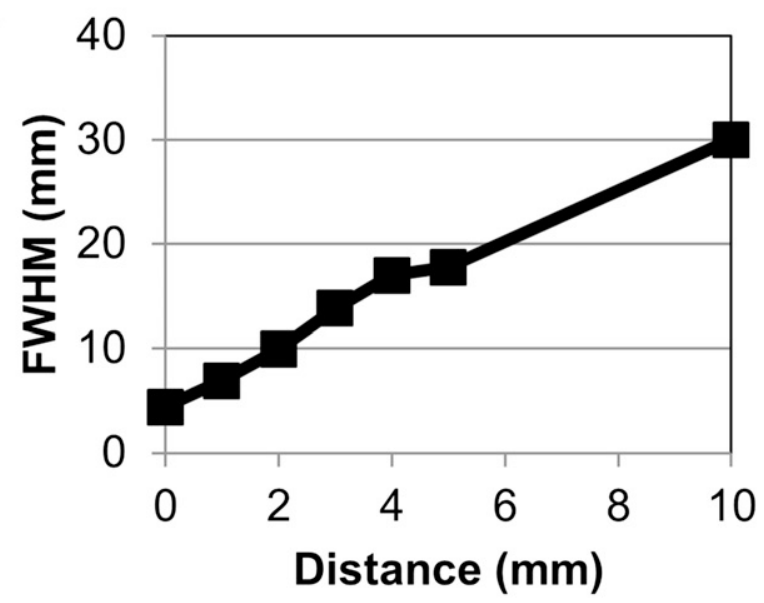

C

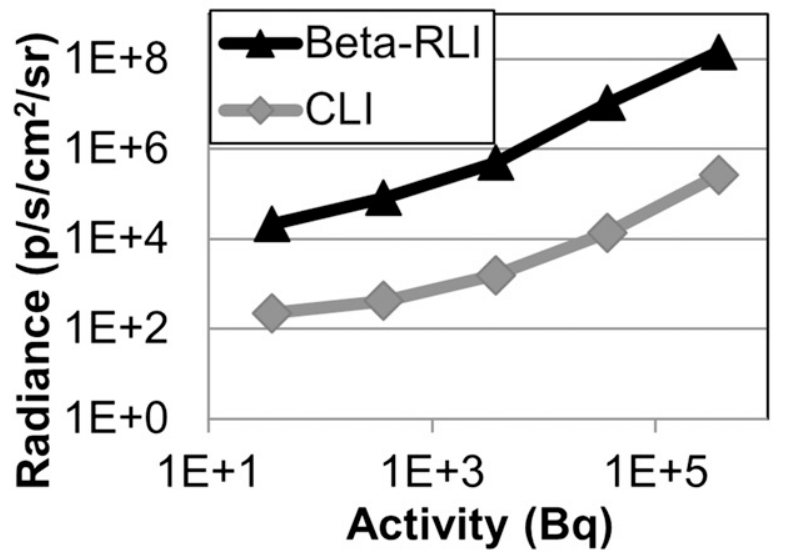

FIGURE 3. Resolution and photon sensitivity analyses. (A) Effect of distance (air and acrylic) between 0.5-mm-diameter capillary tube and scintillator on maximal signal ratio (maximal signal at specified distance/ maximal signal at 1-mm distance for air). (B) Effect of air distance on FWHM resolution. (C) Radiance values of $\beta-R L I$ and $C L I$ for serial ${ }^{18} \mathrm{~F}-\mathrm{FDG}$ dilutions.

$(P=0.55)$ for melanotic lesions. For CLI, 9 of 13 melanotic lesions had an SBR less than 1, despite an SBR greater than 1 among all lesions for $\beta$-RLI. SBR values for RLI (without $\gamma$ rejection) were significantly lower than those for $\beta$-RLI (supplemental data).
Figure 5 shows optical images of a mouse with a $71.0-\mathrm{kBq}(1.92-$ $\mu \mathrm{Ci}) \mathrm{B} 16 \mathrm{~F} 10-\mathrm{Luc}$ tumor after tumor exposure. Although the tumor was clearly visible on $\beta$-RLI, there was negative contrast over the pigmented lesion on CLI. BLI confirms the presence of the tumor. On the other hand, the amelanotic A375 tumor $(31.1 \mathrm{kBq}[0.84 \mu \mathrm{Ci}])$ was clearly visible on CLI, as shown in the left panel of Figure 6.

Serial Resections. Figure 6 shows CLI and $\beta$-RLI of the A375 tumor after partial and full resections. The tumor signals were less apparent after partial resection and almost indiscernible after full resection for both modalities. On the $\beta$-RLI, the high background activity anterior to the tumor bed corresponds to the right knee. This structure may have been closer to the scintillator than the tumor bed, especially after serial restrictions of the initially exophytic tumor.

Table 2 shows SBRs of B16F10-Luc and A375 tumors after stages of serial resections for CLI and $\beta$-RLI. For CLI, partial and full resections of the A375 tumors demonstrated statistically significant decreases in SBR. However, for $\beta$-RLI, only full resections

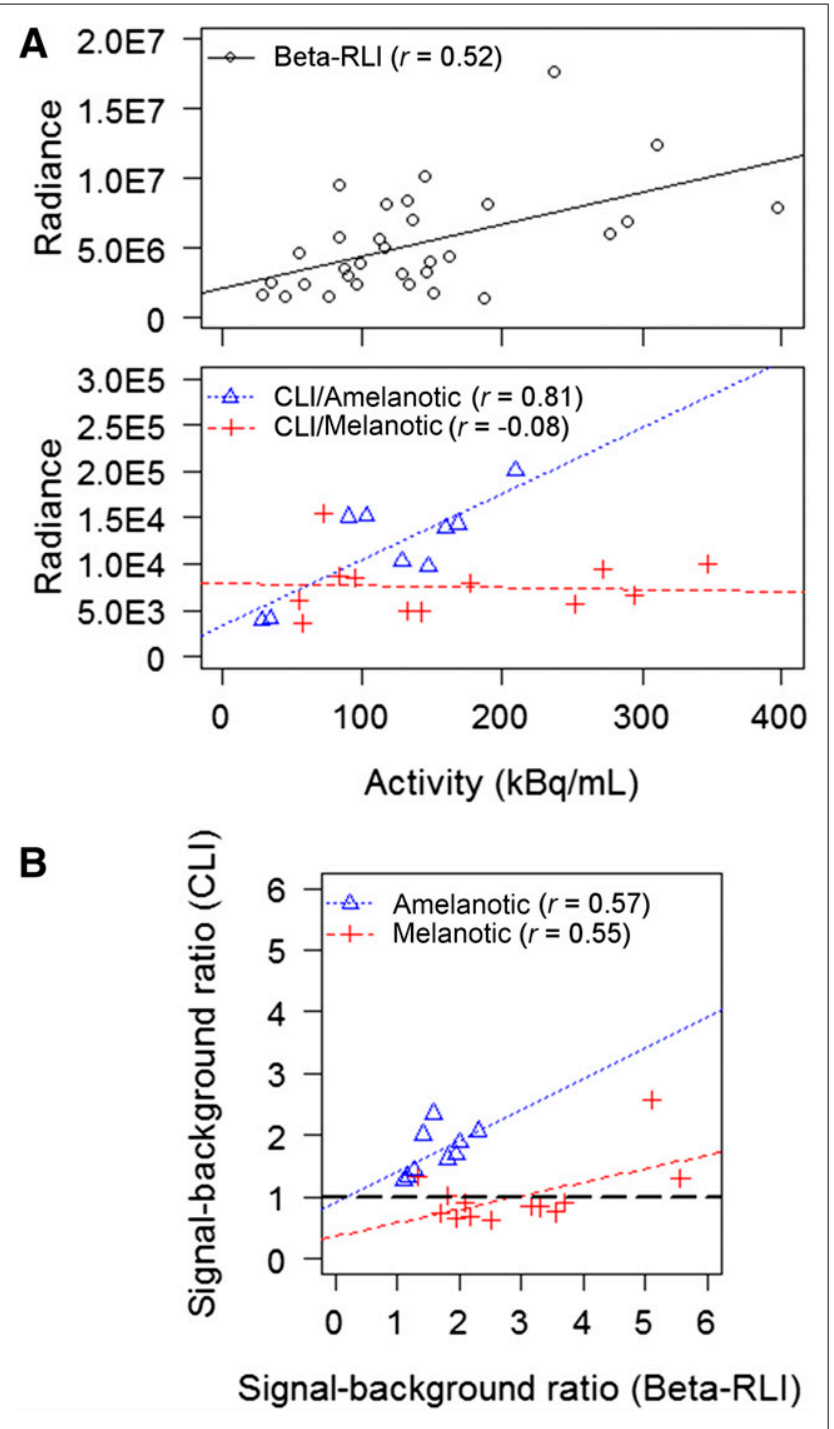

FIGURE 4. (A) Radiance vs. ${ }^{18} \mathrm{~F}-\mathrm{FDG}$ activity $(\mathrm{kBq} / \mathrm{mL})$ for $\beta$-RLI among all tumors, CLI for amelanotic (A375 and UMSCC1-Luc) tumors, and CLI for melanotic (B16F10-Luc) tumors. Radiance has units ( $\left.\mathrm{p} / \mathrm{s} / \mathrm{cm}^{2} / \mathrm{sr}\right)$. (B) SBRs between $\mathrm{CLI}$ and $\beta-\mathrm{RLI}$ for amelanotic and melanotic tumors. 


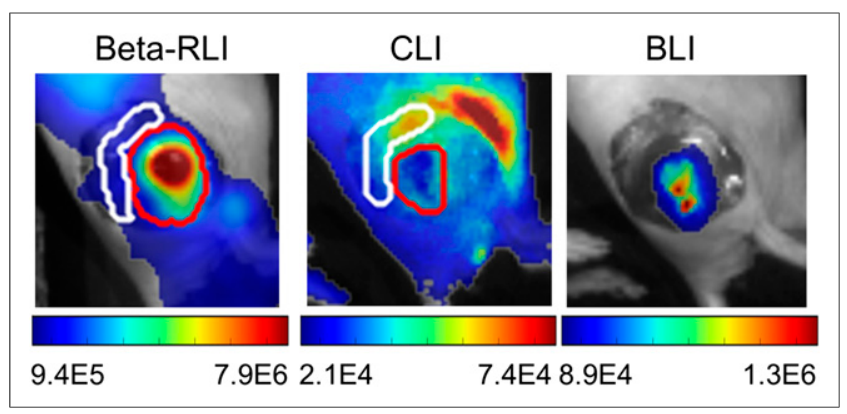

FIGURE 5. $\beta-R L I, C L I$, and BLI of a melanotic B16F10-Luc tumor with $71.0 \mathrm{kBq}(1.92 \mu \mathrm{Ci})$ of ${ }^{18} \mathrm{~F}-\mathrm{FDG}$ by PET after tumor exposure. For $\beta-\mathrm{RLI}$ and CLI, red contours encircle the tumor. White contours encircle the background. SBRs are 3.6 for $\beta-R L I$ and 0.7 for the CLI.

of the A375 and B16F10-Luc tumors demonstrated statistically significant SBR declines ( $P<0.05$ based on 1 -sided $t$ tests).

Residual Disease After Full Resection. All 10 mice that underwent full resections of B16F10-Luc tumors had residual disease based on BLI. The median SBR was 1.0 (minimum, 0.8; maximum, 1.6). Four of 10 tumors (40\%) had an SBR greater than 1.2 and appeared discernible from background in Figure 7. The other 6 tumors were not easily discernible.

\section{DISCUSSION}

In this study, we evaluated a method for the functional imaging of ${ }^{18} \mathrm{~F}$ called $\beta$-RLI. This method incorporates scintillator-based RLI with a $\gamma$-rejection strategy to preferentially image $\beta$ particles. We then performed a comparative evaluation of $\beta$-RLI with CLI. Using in vitro experiments, we showed that $\beta$-RLI has 560 -foldgreater photon sensitivity, which may allow for shorter acquisition times and less stringent requirements regarding ambient lighting intraoperatively. However, $\beta$-RLI also exhibited poorer spatial resolution (FWHM, $4.4 \mathrm{~mm}$, vs. $1 \mathrm{~mm}$ for CLI), limited depth penetration secondary to the positron range of ${ }^{18} \mathrm{~F}(2.4 \mathrm{~mm}$ in water), and declines in both signal intensity and spatial resolution with increasing distance between the object and scintillator.

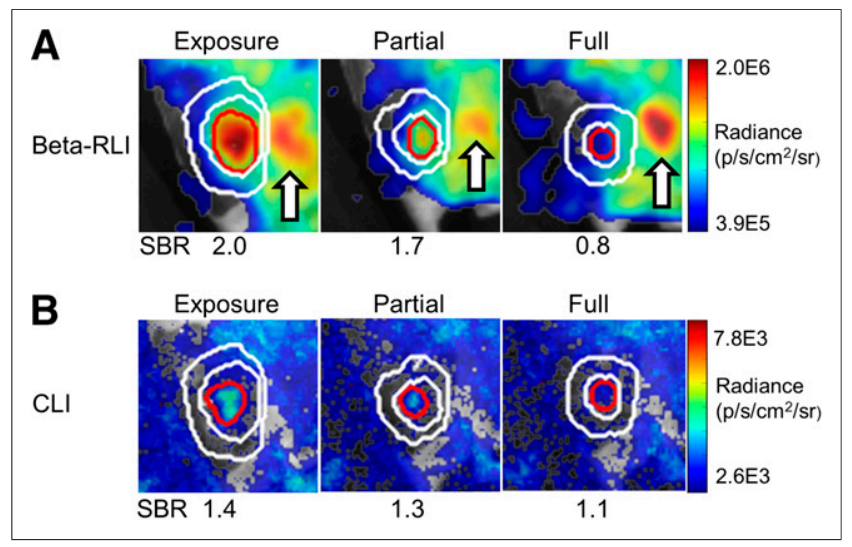

FIGURE 6. $\beta$-RLI and CLI of amelanotic A375 tumor with $31.1 \mathrm{kBq}$ $(0.84 \mu \mathrm{Ci})$ of ${ }^{18} \mathrm{~F}-\mathrm{FDG}$ by PET after serial resections. Serial resections included tumor exposure, partial resection, and full resection. For $\beta-R L I$ and $\mathrm{CLI}$, red contours encircle tumor. White contours encircle background. White arrows point to high signal from mouse knee, which may have been closer to scintillator than tumor bed.
TABLE 2

SBRs for Amelanotic A375 $(n=5)$ Tumors and Melanotic B16F10-Luc $(n=5)$ Tumors After Tumor Exposure, Partial Resection, and Full Resection

\begin{tabular}{|c|c|c|c|c|}
\hline \multirow[b]{2}{*}{ Tumor type } & \multicolumn{2}{|c|}{ CLI } & \multicolumn{2}{|c|}{$\beta-R L I$} \\
\hline & Mean & SD & Mean & SD \\
\hline \multicolumn{5}{|l|}{ A375 } \\
\hline Exposure & 1.8 & 0.4 & 2.0 & 0.2 \\
\hline Partial & $1.5^{\star}$ & 0.2 & 1.8 & 0.2 \\
\hline Full & $1.0^{\star}$ & 0.2 & $1.3^{*}$ & 0.4 \\
\hline \multicolumn{5}{|l|}{ B16F10 } \\
\hline Exposure & 0.8 & 0.2 & 2.3 & 0.2 \\
\hline Partial & NA & NA & 2.0 & 0.2 \\
\hline Full & NA & NA & $1.2^{*}$ & 0.1 \\
\hline
\end{tabular}

*Statistically significant declines in tumor SBR after partial and full resections, based on 1 -sided $t$ test $(P<0.05)$.

Using in vivo mouse models, we demonstrated statistically significant correlations between ${ }^{18} \mathrm{~F}-\mathrm{FDG}$ activity and radiance for amelanotic lesions using $\beta$-RLI and CLI but not for melanotic lesions using CLI. Furthermore, 9 of 13 melanotic lesions had an SBR less than 1 for CLI, despite an SBR greater than 1 among all lesions for $\beta$-RLI. These results suggest that Cerenkov photons were absorbed by melanin. However, $\beta$-RLI often exhibited high background signal after resection, especially for surrounding structures closer to the scintillator (Fig. 6). Furthermore, CLI may provide a more quantitative assessment of tumor burden, as demonstrated by the statistically significant declines in SBR after both partial and full resections of the A375 tumors. Lastly, $\beta$-RLI is not suitable for imaging microscopic disease, as only 4 of 10 B16F10-Luc tumors with microscopic residual disease had an SBR greater than 1.2. Although CLI was not evaluated in this manner, its photon sensitivity is likely insufficient for evaluating microscopic disease (supplemental data).

The imaging properties for $\beta$-RLI are quite similar to those for $\beta$ probes/cameras. With respect to photon sensitivity, a $\beta$ prototype using a phoswich detector for electronic $\gamma$ rejection exhibited lower photon sensitivity $(2.5-14.0 \mathrm{cps} / \mathrm{kBq}$ depending on collimation) (14) than this $\beta$-RLI system (37.3 cps/kBq). However, $\beta$ prototypes using the $\gamma$-subtraction technique that was adapted for $\beta$-RLI demonstrated a greater photon sensitivity $(>100 \mathrm{cps} / \mathrm{kBq})(12,13,16)$. $\beta$ prototypes have a similar spatial resolution $(1.6-5 \mathrm{~mm})(13,15,16)$ and limited depth penetration (16). Furthermore, $\beta$ probes also exhibit decreasing signal intensity and spatial resolution with increasing object-scintillator distance $(14,15)$.

Over the past 2 decades, $\beta$ probes have undergone intensive experimental and clinical evaluations for ${ }^{18} \mathrm{~F}-\mathrm{FDG}$-guided surgery. Experimental studies suggest that $\beta$ probes offer real-time localization of tumor deposits superior to that of $\gamma$ probes $(24,25)$. In addition, clinical studies for the intraoperative tumor localization of melanomas $(13)$ and other cancers $(15,26)$ have yielded promising results.

Given the parallels between $\beta$-RLI and $\beta$ probes/cameras, we would expect ${ }^{18}$ F-FDG-guided surgery to be the main clinical application for $\beta$-RLI. However, $\beta$-RLI has potential advantages 


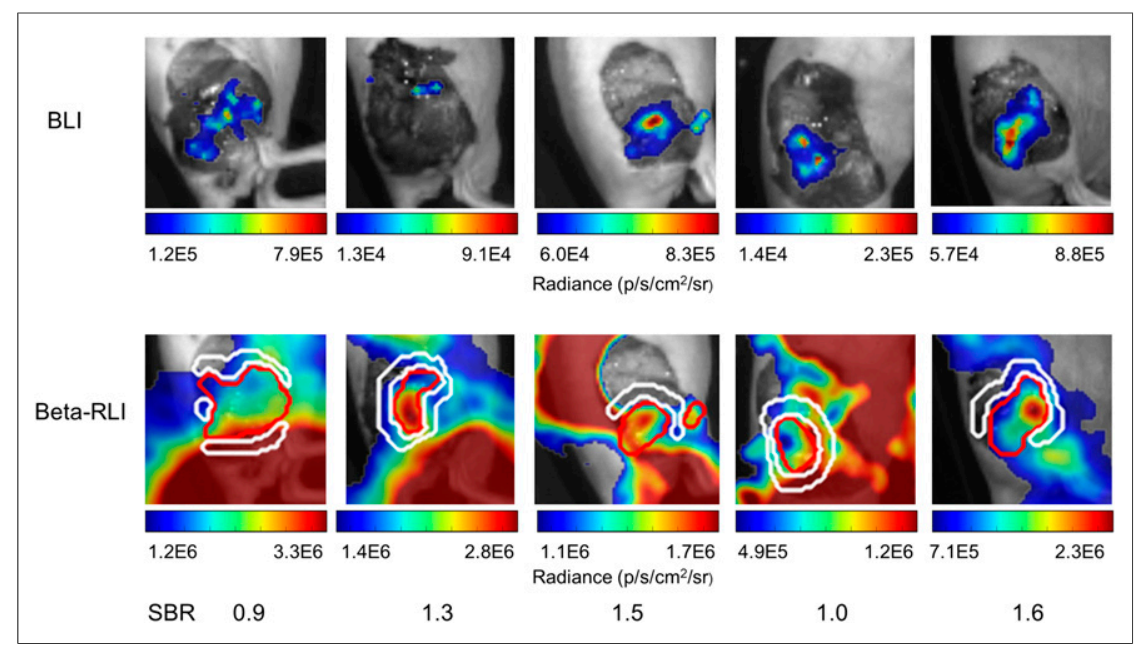

FIGURE 7. BLI and $\beta-R L I$ after full tumor resections for 5 mice with B16F10-Luc tumors. For $\beta-R L I$, red contours encircle tumor. White contours encircle background. Each $\beta$ image is scaled between minimum and maximum values within red contour to accentuate signal contrast within tumor bed. As such, background may appear saturated. High signal over tail region may be secondary to ${ }^{18} \mathrm{~F}-\mathrm{FDG}$ accumulation from tail vein injection or urine. Image on very right corresponds to mouse in Figure 5. depth penetration, high background, and the need for acquiring 2 images. Further engineering developments are needed to realize the full clinical potential of this modality.

\section{DISCLOSURE}

The costs of publication of this article were defrayed in part by the payment of page charges. Therefore, and solely to indicate this fact, this article is hereby marked "advertisement" in accordance with 18 USC section 1734. Funding for this work was provided by the RSNA research resident/ fellow grant (RR1326), the Jacob Haimson endowed fellowship fund, the National Institutes of Health, the Stanford Department of Radiation Oncology, and the Key Program of National Natural Science Foundation of China (81230033). No other potential conflict of interest relevant to this article was reported. over $\beta$ probes/cameras. First, $\beta$-RLI has optical image overlay, which can allow the surgeon to correlate areas of increased activity with anatomic features. Second $\beta$-RLI can provide a large imaging field of view for efficient functional mapping of the operative bed.

There are other methods for improving the sensitivity of CLI without scintillators. Down-conversion involves using nanoparticles to shift the wavelength of Cerenkov light for better tissue penetration (27). However, most nanoparticles are exogenous agents that have not been approved by the Federal Drug Administration for human use. High-energy $\beta$-emitting radionuclides such as ${ }^{90} \mathrm{Y}$ exhibit sensitivity superior to ${ }^{18} \mathrm{~F}$. However, ${ }^{90} \mathrm{Y}$ requires conjugation to peptides, such as arginine-glycine-aspartate, for tumor targeting (28).

Future research developments may revolve around scintillator design for improving the acquisition and image quality of RLI. First, a single-acquisition $\beta$-RLI technique could be realized by attaching a stainless steel grid onto a scintillating sheet. Because each image contains information from both RLI and block-RLI, interpolation algorithms could be used to recover the missing portions of these images before image subtraction for $\gamma$ rejection, possibly allowing for decreased acquisition time and reduced image noise. Furthermore, $\gamma$ rejection may be improved, because the scintillator and object often shift between RLI and block-RLI acquisitions. Second, spatial resolution may be improved through the incorporation of collimation (14) but at the expense of photon sensitivity. Third, flexible scintillators (29) may improve sensitivity by maximizing the contact between the tumor and scintillator and minimizing the effect of distance variations between the tumor and scintillator on signal intensities.

\section{CONCLUSION}

$\beta$-RLI can produce high-contrast functional images of both amelanotic and melanotic tumors in a shorter time frame than CLI. $\beta$-RLI limitations include poor spatial resolution, limited

\section{ACKNOWLEDGMENTS}

We acknowledge the Stanford Small Animal Imaging Facility and the Stanford Radiochemistry Facility for providing the resources required for this study.

\section{REFERENCES}

1. Robertson R, Germanos MS, Li C, Mitchell GS, Cherry SR, Silva MD. Optical imaging of Cerenkov light generation from positron-emitting radiotracers. Phys Med Biol. 2009;54:N355-N365.

2. Spinelli AE, D'Ambrosio D, Calderan L, Marengo M, Sbarbati A, Boschi F. Cerenkov radiation allows in vivo optical imaging of positron emitting radiotracers. Phys Med Biol. 2010;55:483-495.

3. Liu H, Ren G, Miao Z, et al. Molecular optical imaging with radioactive probes. PLoS One. 2010;5:e9470.

4. Xu Y, Chang E, Liu H, Jiang H, Gambhir SS, Cheng Z. Proof-of-concept study of monitoring cancer drug therapy with Cerenkov luminescence imaging. $J$ Nucl Med. 2012;53:312-317.

5. Robertson R, Germanos MS, Manfredi MG, Smith PG, Silva MD. Multimodal imaging with ${ }^{18}$ F-FDG PET and Cerenkov luminescence imaging after MLN4924 treatment in a human lymphoma xenograft model. J Nucl Med. 2011;52:1764-1769.

6. Holland JP, Normand G, Ruggiero A, Lewis JS, Grimm J. Intraoperative imaging of positron emission tomographic radiotracers using Cerenkov luminescence emissions. Mol Imaging. 2011;10:177-186, 1-3.

7. Liu $\mathrm{H}$, Carpenter $\mathrm{CM}$, Jiang $\mathrm{H}$, et al. Intraoperative imaging of tumors using Cerenkov luminescence endoscopy: a feasibility experimental study. J Nucl Med. 2012;53:1579-1584.

8. Spinelli AE, Ferdeghini M, Cavedon C, et al. First human Cerenkography. J Biomed Opt. 2013;18:020502.

9. Thorek DLJ, Riedl CC, Grimm J. Clinical Cerenkov luminescence imaging of ${ }^{18}$ F-FDG. J Nucl Med. 2014;55:95-98.

10. Hu H, Cao X, Kang F, et al. Feasibility study of novel endoscopic Cerenkov luminescence imaging system in detecting and quantifying gastrointestinal disease: first human results. Eur Radiol. 2015;25:1814-1822.

11. Scintillation properties. Lawrence Berkeley National laboratory website. http://scintillator.lbl.gov/. Accessed July 30, 2015.

12. Daghighian F. Intraoperative beta probe: a device for detecting tissue labeled with positron or electron emitting isotopes during surgery. Med Phys. 1994;21: $153-157$.

13. Franc BL, Mari C, Johnson D, Leong SP. The role of a positron-and high-energy gamma photon probe in intraoperative localization of recurrent melanoma. Clin Nucl Med. 2005;30:787-791. 
14. Yamamoto S, Higashi T, Matsumoto K, Senda M. Development of a positronimaging detector with background rejection capability. Ann Nucl Med. 2006;20: $655-662$.

15. Piert M, Burian M, Meisetschläger G, et al. Positron detection for the intraoperative localisation of cancer deposits. Eur J Nucl Med Mol Imaging. 2007;34: 1534-1544.

16. Bogalhas F, Charon Y, Duval M-A, et al. Development of a positron probe for localization and excision of brain tumours during surgery. Phys Med Biol. 2009;54:4439-4453.

17. Spinelli AE, Boschi F. Novel biomedical applications of Cerenkov radiation and radioluminescence imaging. Phys Med. 2015;31:120-129.

18. Boschi F, Spinelli AE, D'Ambrosio D, Calderan L, Marengo M, Sbarbati A. Combined optical and single photon emission imaging: preliminary results. Phys Med Biol. 2009;54:L57-L62.

19. Spinelli AE, Meo SL, Calandrino R, Sbarbati A, Boschi F. Optical imaging of Tc99m-based tracers: in vitro and in vivo results. J Biomed Opt. 2011;16:116023.

20. Boschi F, Meo SL, Rossi PL, Calandrino R, Sbarbati A, Spinelli AE. Optical imaging of alpha emitters: simulations, phantom, and in vivo results. J Biomed Opt. 2011;16:126011.

21. Pratx G, Chen K, Sun C, et al. Radioluminescence microscopy: measuring the heterogeneous uptake of radiotracers in single living cells. PLoS One. 2012;7: e46285.
22. Zaman RT, Kosuge H, Pratx G, Carpenter C, Xing L, McConnell MV. Fiber-optic system for dual-modality imaging of glucose probes ${ }^{18} \mathrm{~F}-\mathrm{FDG}$ and 6-NBDG in atherosclerotic plaques. PLoS One. 2014;9:e108108.

23. Yushkevich PA, Piven J, Hazlett HC, et al. User-guided 3D active contour segmentation of anatomical structures: significantly improved efficiency and reliability. Neuroimage. 2006;31:1116-1128.

24. Strong VE, Galanis CJ, Riedl CC, et al. Portable PET probes are a novel tool for intraoperative localization of tumor deposits. Ann Surg Innov Res. 2009;3:2

25. Garcia-Parra R, Clinthorne N, Wang L, Picchio M, Piert M. Performance of betaand high-energy gamma probes for the detection of cancer tissue in experimental surgical resection beds. Ann Nucl Med. 2011;25:486-493.

26. Yang Y, Biswal NC, Wang T, et al. Potential role of a hybrid intraoperative probe based on OCT and positron detection for ovarian cancer detection and characterization. Biomed Opt Express. 2011;2:1918-1930.

27. Dothager RS, Goiffon RJ, Jackson E, Harpstrite S, Piwnica-Worms D. Cerenkov radiation energy transfer (CRET) imaging: a novel method for optical imaging of PET Isotopes in biological systems. PLoS One. 2010;5:e13300.

28. Carpenter CM, Ma X, Liu H, et al. Cerenkov luminescence endoscopy: improved molecular sensitivity with $\beta$-emitting radiotracers. J Nucl Med. 2014;55:19051909.

29. Jenkins CH, Naczynski DJ, Yu S-JS, Xing L. Monitoring external beam radiotherapy using real-time beam visualization. Med Phys. 2015;42:5-13. 